Supporting Information

\title{
Fluorescence Products from Terrylenediimide (TDI) with Singlet Oxygen: Red, Green and Near-Infrared Emission
}

Hongmei Zhao, ${ }^{a}$ Kunhui Liu, ${ }^{b}$ Di Song ${ }^{a}$, and Hongmei Su* ${ }^{a, b}$

${ }^{a}$ Beijing National Laboratory for Molecular Sciences (BNLMS), Institute of

Chemistry, Chinese Academy of Sciences, Beijing 100190, P. R. China.

${ }^{\mathrm{b}}$ College of Chemistry, Beijing Normal University, Beijing 100875, P.R.China

AUTHOR INFORMATION

Corresponding Author

*Email: hongmei@iccas.ac.cn Tel: +86-10-62562837 


\section{Complete list of supporting information}

Figure S1-The optimized geometries of reactants, intermediates, transition states and products obtained at the level of restricted $\mathrm{B} 3 \mathrm{LYP} / 6-31 \mathrm{G}^{*}$ for closed shell species and unrestricted broken symmetry (U)B3LYP/6-31G* for open shell diradical species. Bond lengths are indicated in angstrom.

Figure S2- The molecular structure of TS1, TS4 and TS7.

Table S1-Product absorption peak wavelength $(\lambda)$ and oscillator strengths (f) obtained at the TD-B3LYP/def-SVP level.

Table S2-Fluorescence peak wavelength $(\lambda)$ and oscillator strengths (f) obtained at the TD-B3LYP/def-SVP level, for the possible secondary products resulted from the multiple attack of ${ }^{1} \mathrm{O}_{2}$ to TDI. 
Figure S1. The optimized geometries of reactants, intermediates, transition states and products obtained at the level of restricted $\mathrm{B} 3 \mathrm{LYP} / 6-31 \mathrm{G}^{*}$ for closed shell species and unrestricted broken symmetry (U)B3LYP/6-31G* for open shell diradical species. Bond lengths are indicated in angstrom. The naphthalene units subject to ${ }^{1} \mathrm{O}_{2}$ addition are labeled with blue balls. Red balls represent oxygen atoms.

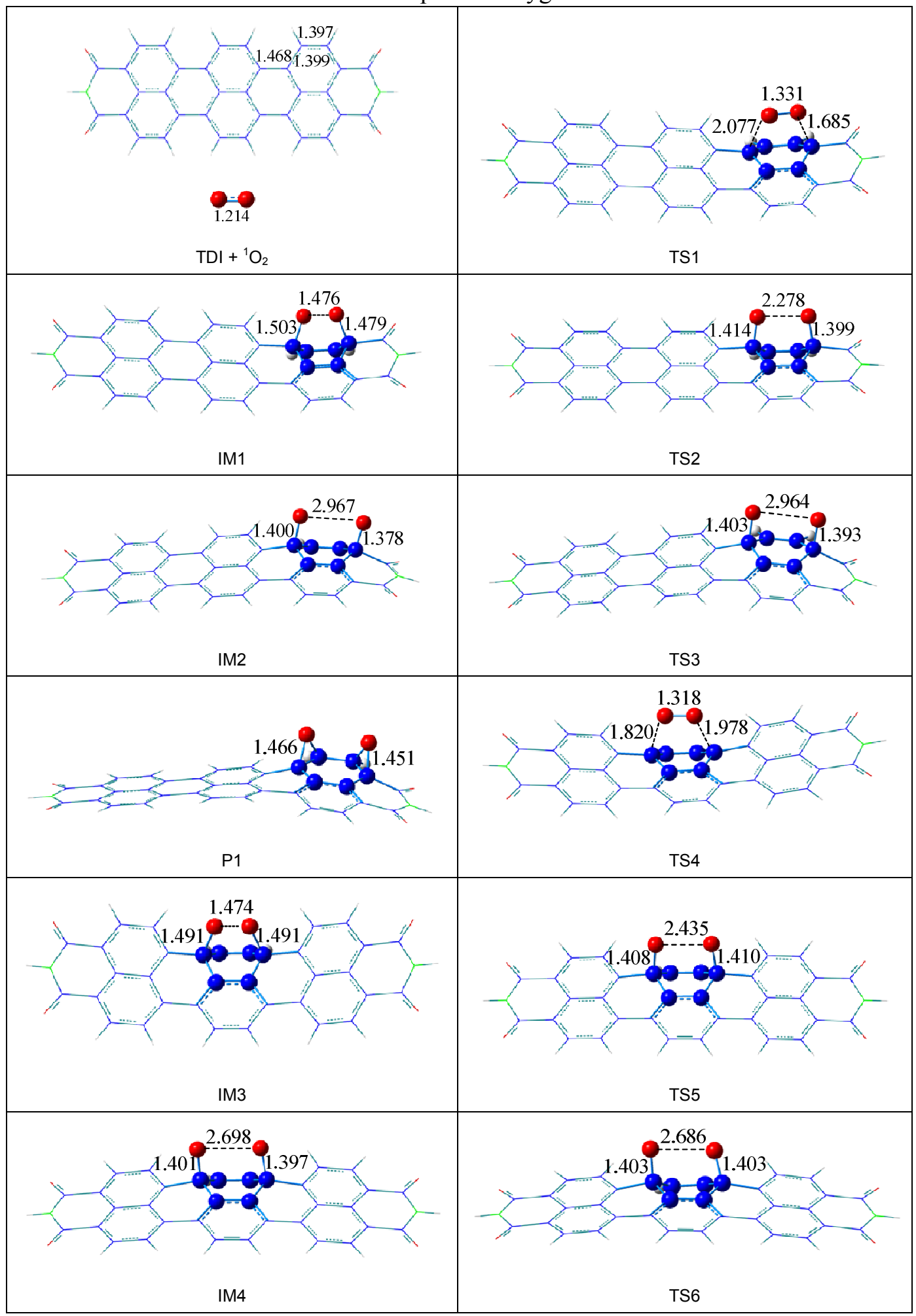




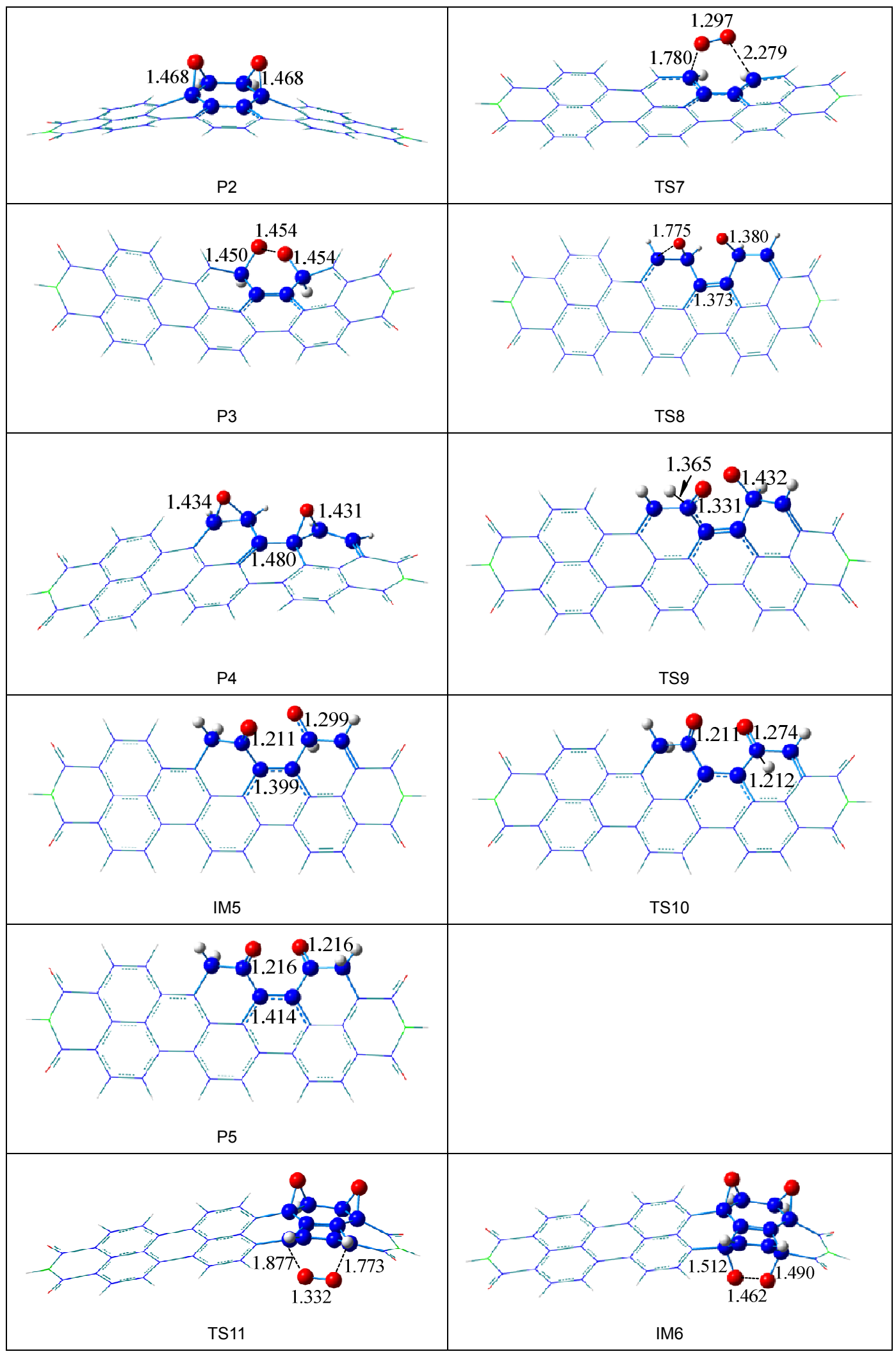




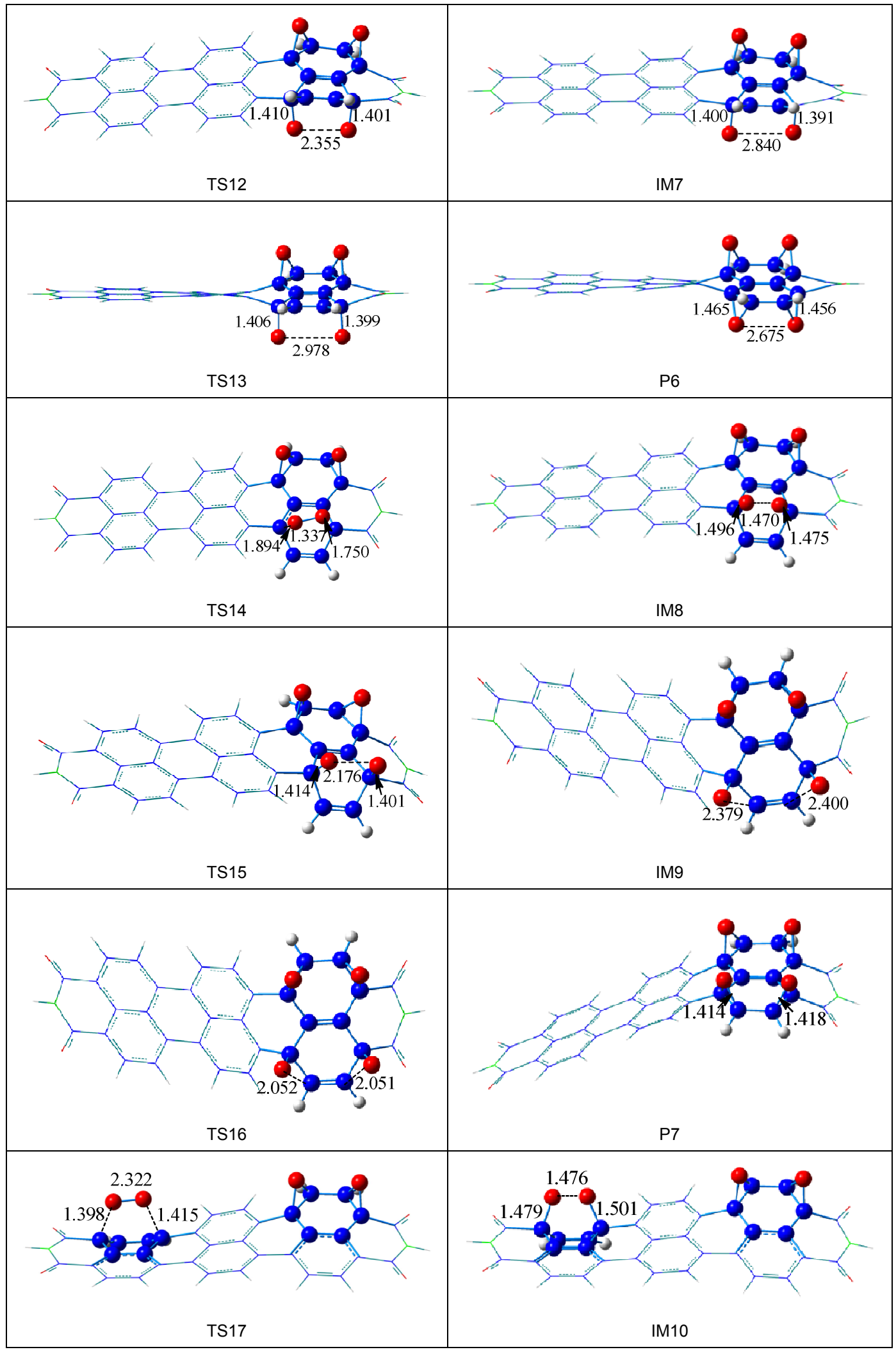




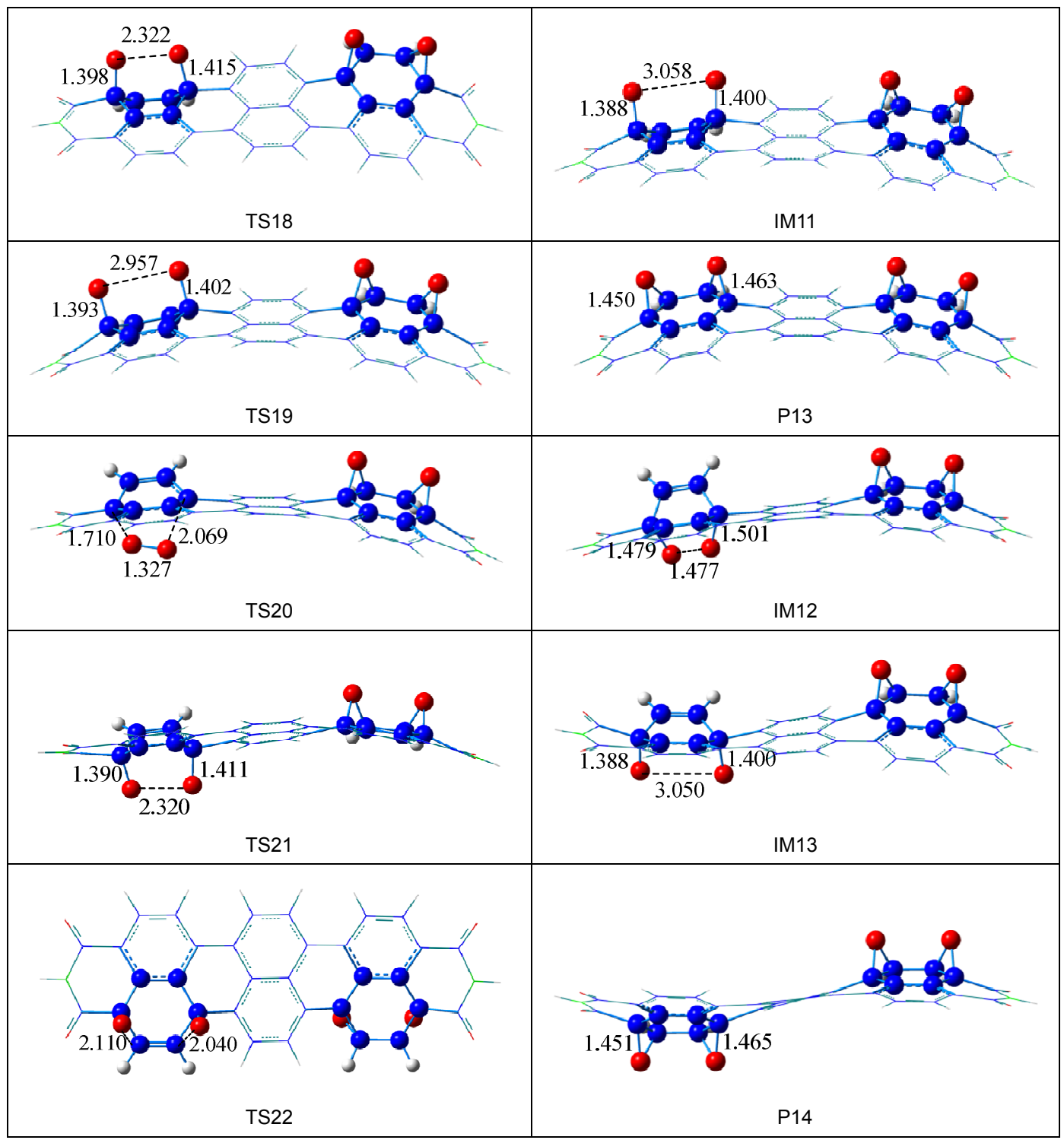

Figure S2. The molecular structure of TS1 in pathway 1, TS4 in pathway 2 and TS7 in pathway 3 .
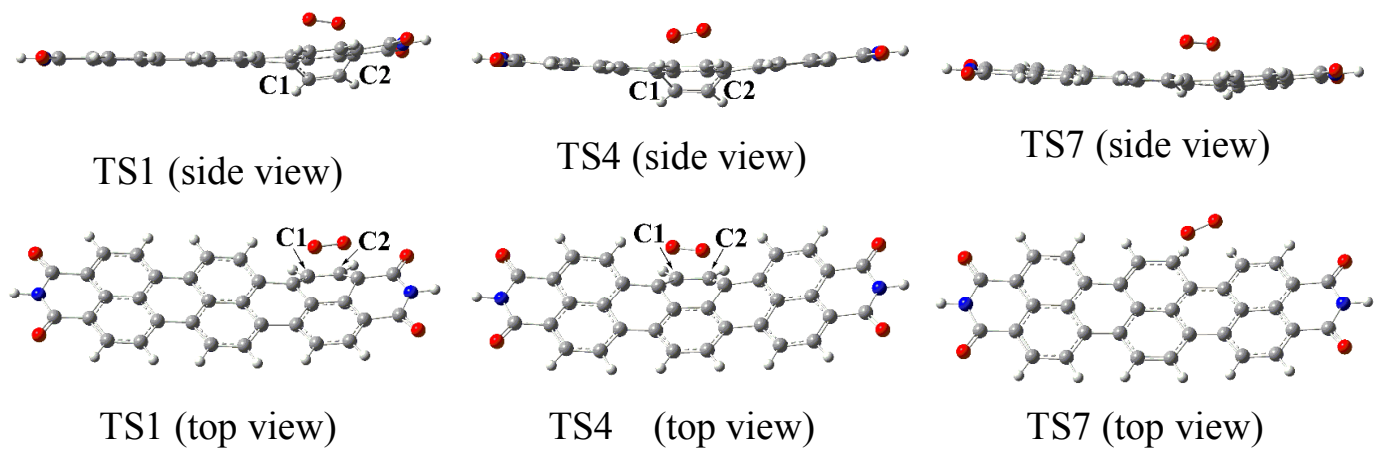
Table S1. Product absorption peak wavelength $(\lambda)$ and oscillator strengths (f) obtained at the TD-B3LYP/def-SVP level.

\begin{tabular}{|c|c|c|}
\hline product & $\lambda / \mathrm{nm}$ & $f$ \\
\hline P1 & 535 & 0.88 \\
\hline P2 & 456 & 0.82 \\
\hline P3 & 452 & 0.31 \\
\hline P4 & 710 & 0.71 \\
\hline P5 & 902 & 0.73 \\
\hline
\end{tabular}


Table S2. Fluorescence peak wavelength $(\lambda)$ and oscillator strengths (f) obtained at the TD-B3LYP/def-SVP level, for the possible secondary products resulted from the multiple attack of ${ }^{1} \mathrm{O}_{2}$ to TDI. The naphthalene units subject to ${ }^{1} \mathrm{O}_{2}$ addition are labeled with blue balls. Red balls represent oxygen atoms.

\begin{tabular}{|l|l|l|l|}
\hline & $1 / \mathrm{mm}$ & \\
\hline \\
\hline
\end{tabular}




\begin{tabular}{|l|l|l|}
\hline & \\
\hline
\end{tabular}

